\title{
INTERSECTIONS OF COMBINATORIAL BALLS AND OF EUCLIDEAN SPACES
}

\author{
BY L. C. GLASER \\ Communicated by O. G. Harrold, August 9, 1965
}

1. Introduction. Poenaru [4] and Mazur [3] gave the first examples of pseudo 4-cells whose products with the unit interval were combinatorial 5-cells. Curtis [1] and Glaser [2] gave similar examples for $n \geqq 5$. In addition, it was shown in [2], that, for $n \geqq 5$, the pseudo $n$-cell $M^{n}$ could be expressed as the union of two combinatorial $n$ cells whose intersection is also a combinatorial $n$-cell. Unfortunately, the techniques used in [2] gave no hope of lowering the result to $n=4$.

The purpose of this announcement is to give another example of a pseudo 4-cell $W$ with the property that $W \times I \approx I^{5}$, but in addition $W$ can be expressed as the union of two combinatorial 4-cells whose intersection is also a combinatorial 4-cell. This also gives an example of two Euclidean 4-spaces intersecting in an Euclidean 4-space so that the union is not topologically $E^{4}$.

Our techniques and terminology basically follow that found in [6], [7].

2. Construction. Let us consider a figure eight expressed as the union of four line segments $\alpha, \beta, \gamma$, and $\delta$ and three vertices $a, b$, and $c$ as indicated in Figure 1. Let $K$ be the contractible noncollapsible 2complex formed by attaching two disks to the figure eight by the formula $\beta \gamma \gamma^{-1} \delta^{-1} \delta \alpha$ and $\delta \alpha \alpha^{-1} \beta^{-1} \beta \gamma$.

Let $T$ be a solid two-holed 3-dimensional torus in $E^{3}$. The pseudo 4-cell $W$ will be formed by attaching two 2-handles to the boundary of $T \times[0,1]$ along the curves $\Gamma_{1}$ and $\Gamma_{2}$ embedded in $\operatorname{int}(T \times\{1\}) \subset T$ $\times[0,1]$ as indicated in Figure 1 .

LEMMA 1. W can be considered as a regular neighborhood of a combinatorial embedding of $K$ in $W$.

Proof. We can obtain a copy of $K$ by "pushing" $\Gamma_{1} \cup \Gamma_{2}$ down to a core of $T \times[0,1]$ in an appropriate manner and then considering the point set consisting of the trace of this "pushing" plus the two disks gotten from adding the two 2-handles. The remainder of the proof consists of showing how we can triangulate $W$ so that $K$ is a subcomplex and $W \searrow K$. The techniques used here are similar to those of [7]. 
Theorem 1. $\pi_{1}(\mathrm{Bd} W) \neq 1$.

Proof. $\pi_{1}(\mathrm{Bd} W)$ has the following group presentation: generators: $a, b, x, y$ and $z$ relations:

I. $\quad \bar{a} \bar{x} \bar{a} \bar{y} \bar{a}$ y $a x a x \bar{a} \bar{x} \bar{a} \bar{y} a$ y $a x a \bar{x} \bar{a} \bar{x} \bar{a} \bar{y} a$ y $a x=1$

II. $\quad \bar{a} \bar{x} \bar{a} \bar{y} a$ y $a x a \bar{x} \bar{a} z a x \bar{a} \bar{x} \bar{a} \bar{y} \bar{a}$ y $a x a \bar{x}=1$

III. $\bar{y} b y z \bar{b} \bar{z} \bar{y} b y \bar{b} y z b \bar{z} \bar{y} b y z \bar{b} \bar{z} \bar{y} b=1$

IV. $\bar{y} \bar{b} y z b \bar{z} \bar{y} b y z \bar{z} \bar{y} \bar{a} y a x b \bar{z} b y z b \bar{z} \bar{y} \bar{b} y z \bar{b} \bar{z} \bar{y} b=1$

$\Gamma_{1}: \quad \bar{x} \bar{a} \bar{y} \bar{x} \bar{a} \bar{y} \bar{a}$ y $a x a x \bar{a}=1$

$\Gamma_{2}: \quad \bar{z} \bar{y} b y b y z b \bar{z} \bar{y} b=1$

$\mathrm{K}_{1}: \quad x \bar{a} \bar{x} \bar{a} \bar{y} a$ y $a x a \bar{x} \bar{a} \bar{b}=1$

$\mathrm{K}_{2}: \quad \bar{a} b y z b \bar{z} \bar{y} b y z b \bar{z} \bar{y}=1$
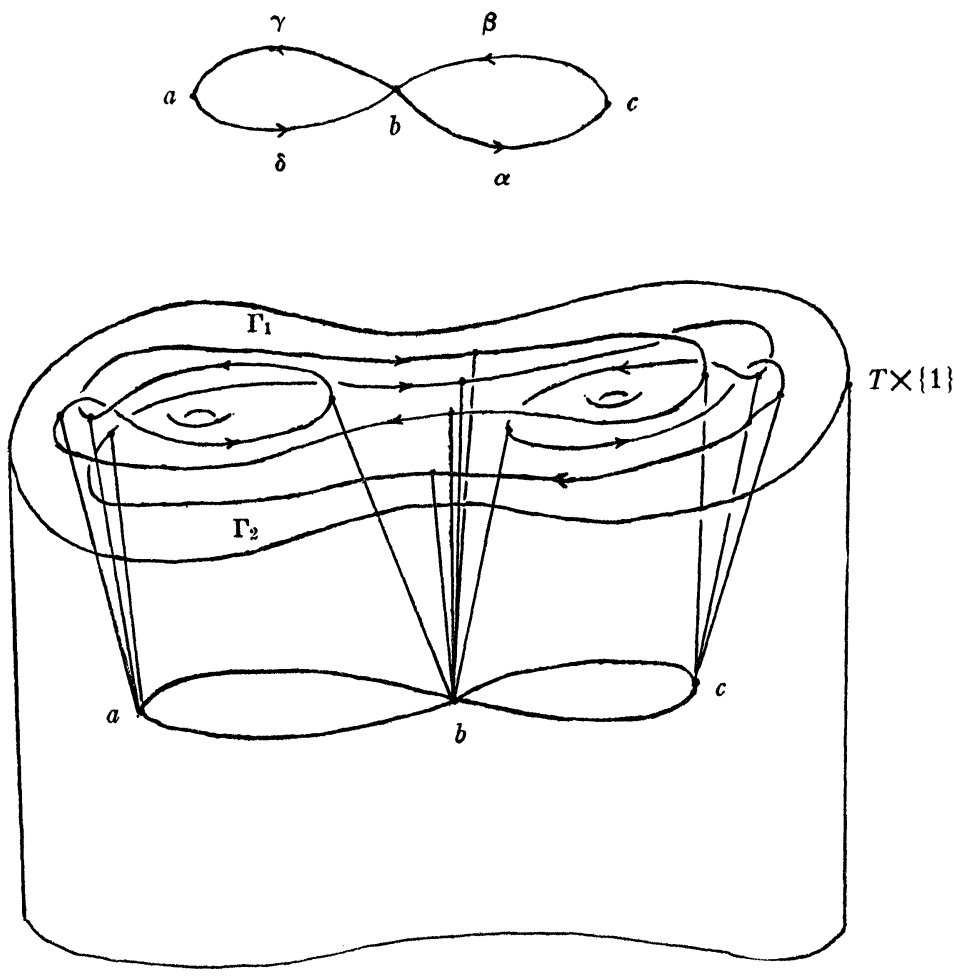

FIGURE 1

This group can be shown to have a nontrivial representation in $P_{5}$ 
by letting $a \rightarrow(14253), b \rightarrow(12543), x \rightarrow(14352), y \rightarrow(12453)$ and $z \rightarrow(14523)$.

\section{Main results.}

LeMma 2. Suppose $K$ is a contractible 2-subcomplex in the interior of a combinatorial 4-manifold $M$ and $W$ is a regular neighborhood of $K$ in $M$. If $K$ can be combinatorially embedded in $E^{3}$, then $W$ can be embedded in $E^{4}$ and $W \times I \approx I^{5}$.

The proof of this lemma follows from results of [1] and [5].

THEOREM 2. There exists a pseudo 4-cell $W \neq I^{4}$ such that $W \subset E^{4}$, $W \times I \approx I^{5}$ and $W \approx X \cup Y$, where $X \approx Y \approx X \cap Y \approx I^{4}$.

This theorem follows from Lemma 1, Theorem 1, and Lemma 2 and the fact that $K \approx K_{1} \cup K_{2}$ where $K_{1} \searrow 0, K_{2} \searrow 0$ and $K_{1} \cap K_{2} \searrow 0$.

Combining Theorem 2 with results from [2], we get the following two corollaries.

Corollary 1. For $n \geqq 4$ there exist pseudo $n$-cells $W^{n} \neq I^{n}$ such that $W^{n} \times I \approx I^{n+1}$ and $W^{n} \approx X^{n} \cup Y^{n}$, where $X^{n} \approx Y^{n} \approx X^{n} \cap Y^{n} \approx I^{n}$.

COROLlaRy 2. For $n \geqq 3$ there exist open contractible combinatorial $n$-manifolds $0^{n} \neq E^{n}$ such that $0^{n} \approx U^{n} \cup V^{n}$, where $U^{n} \approx V^{n} \approx U^{n} \cap V^{n}$ $\approx E^{n}$.

REMARK. The result for $n=3$ is gotten by considering the complement of a double Fox-Artin arc in $S^{3}$.

The next result says that in some sense the given embeddings are the simplest possible in order to get an example where $\pi_{1}(\operatorname{Bd} W) \neq 1$. Also the linking or local knottedness of $\Gamma_{1}$ or $\Gamma_{2}$ with itself or with each other is irrelevant if the embeddings are "nice" in the opposite ends of $T$.

Each of $\operatorname{lk}(a, K)$ and $\operatorname{lk}(c, K)$ is merely two circles, $C_{1}$ and $C_{2}$ say, joined by an $\operatorname{arc} A$. We will say that the embedding of $K$ in the interior of a combinatorial 4-manifold $M^{4}$ is nice at $a$ if $1 \mathrm{k}(a, K)$ in $1 \mathrm{k}\left(a, M^{4}\right)$ is such that there exists a 2 -sphere in $\operatorname{lk}\left(a, M^{4}\right)$ separating $C_{1}$ and $C_{2}$ and meeting $A$ in a single point $z \in \operatorname{int} A$. Similarly for the vertex $c$.

Theorem 3. Let $K \subset$ int $M^{4}$ and suppose $M^{4} \searrow K$. If the embedding of $K$ is nice at $a$ and $c$, then $M^{4} \approx I^{4}$.

Corollary 3. If $K C$ int $M^{n}(n \geqq 5)$ and $M^{n} \searrow K$, then $M^{n} \approx I^{n}$.

The techniques used in proving Theorem 3 are similar to those used in [7]. The proof of Corollary 3 follows from the proof of Theorem 3 or applying the results of [5]. 


\section{BIBLIOGRAPHY}

1. M. L. Curtis, Cartesian products with intervals, Proc. Amer. Math. Soc. 12 (1961), 819-820.

2. L. C. Glaser, Contractible complexes in $S^{n}$, Proc. Amer. Math. Soc. 16 (1965), (to appear).

3. B. Mazur, A note on some contractible 4-manifolds, Ann. of Math. 73 (1961), 221-228.

4. V. Poenaru, Les decompositions de l'hypercube en produit topologique, Bull. Soc. Math. France 88 (1960), 113-119.

5. T. Price, Equivalence of embeddings of $k$-complexes in Euclidean $n$-space for $n \leqq 2 k+1$, Abstract 614-121, Notices Amer. Math. Soc. 11 (1964), 564.

6. J. H. C. Whitehead, Simplicial spaces, nuclei and m-groups, Proc. London Math. Soc. 45 (1939), 243-327.

7. E. C. Zeeman, On the dunce hat, Topology 2 (1964), 341-358.

RICE UNIVERSITY

\section{EXTREMA CONCERNING POLYGONS IN SPACE-TIME}

BY LOUIS V. QUINTAS AND FRED SUPNICK

Communicated by Victor L. Klee, August 6, 1965

1. Consider an astronomer and his observation field, i.e., the set of observable (light or radio) signal emitting loci of the universe. Let the observation field be ordered by attaching a date to each observable locus indicating the time in the history of the universe that the signal was emitted from its source. Whereas both the astronomer and his observation field age with time, the observations of the astronomer may trace a sequence of loci whose time labels proceed forward or backward in time.

Consider now, a finite set $S$ of events in $L^{n}, n$-dimensional spacetime $^{1}(n \geqq 2)$. A rectilinear world line segment with endpoints in $S$ will be called a rectilinear connection in $S$ and a set of rectilinear connections which form a polygon with vertex set $S$ a polygonal connection of $S$. The clock time of a polygonal connection is defined to be the sum of all the time separations ${ }^{2}$ of its rectilinear connections. A polygonal connection having either the least or the greatest clock time of all possible "circuit states," i.e., all possible polygonal connections of

\footnotetext{
${ }^{1}$ Riemannian $n$-space having fundamental form $\Phi=\left(d x^{1}\right)^{2}+\cdots+\left(d x^{n-1}\right)^{2}$ $-(d t)^{2}$.

2 The time separation of a rectilinear connection with endpoints $E_{u}:\left(x_{u}^{1}, \cdots, x_{u}^{n-1}, t_{u}\right)$ and $E_{v}:\left(x_{v}^{1}, \cdots, x_{v}^{n-1}, t_{v}\right)$ is equal to $\left[\left(t_{v}-t_{v}\right)^{2}-\sum_{i=1}^{n-1}\left(x_{v}^{i}-x_{u}^{i}\right)^{2}\right]^{1 / 2}$ and will be denoted by $s\left(E_{w} E_{v}\right)$.
} 